Meta

Journal des traducteurs

Translators' Journal

\title{
Index français des mots et des sujets traités
}

Volume 42, numéro 4, décembre 1997

URI : https://id.erudit.org/iderudit/003176ar

DOI : https://doi.org/10.7202/003176ar

Aller au sommaire du numéro

Éditeur(s)

Les Presses de l'Université de Montréal

ISSN

0026-0452 (imprimé)

1492-1421 (numérique)

Découvrir la revue

Citer ce document

(1997). Index français des mots et des sujets traités. Meta, 42(4), 754-761.

https://doi.org/10.7202/003176ar d'utilisation que vous pouvez consulter en ligne.

https://apropos.erudit.org/fr/usagers/politique-dutilisation/ 


\section{Index français des mots}

\section{et des sujets traités}

Aduriz, Jose Antonio et Miriam Urkia: La lexicographie assistée par ordinateur. L'expérience $d^{\prime} U_{z e i}, \mathrm{n}^{\circ} 2$, p. 257.

Akakuru, Theanacho A. et Nwanne Mkpa : Traduction et stylistique : une analyse de la traduction d'Arrow of God de Chinua Achebe, $\mathrm{n}^{\circ} 4$, p. 630 .

Almeida, Marina de, Margreet de Rooij, Marie-Hélène Lattaro, Mayra Parra, Justine Ravonihasindaza, Hélène Saulnier et Cécile Bruneau : Je construis ma maison. Etude lexico-terminologique de la construction, $\mathrm{n}^{\circ} 4$, p. 685 .

Alphabet manuel, $n^{\circ}$ 3. p. 488.

Analyse de l'autonomie, ${ }^{\circ} 1$, p. 48.

Analyse du sens d'un concept, $\mathrm{n}^{\circ} 2$, p. 240.

Analyse en contexte, $n^{\circ} 1$, p. 48.

Analyse morphosyntaxique, $\mathrm{n}^{\circ} 2$, p. 356.

Analyse syntaxique, $n^{\circ} 2$, p. 357 .

Anglais contemporain non standard, $\mathrm{n}^{\circ} 1$, p. 55 .

Arabe, système verbal, $n^{\circ} 2$, p. 317.

Arabe, transcription de l', $\mathrm{n}^{\circ}$ 2, p. 292.

Architecture client-serveur, $\mathrm{n}^{\circ} 2$, p. 284

Arguments, $\mathrm{n}^{\circ} 1, \mathrm{p} .147$.

BALNEO, $\mathrm{n}^{\circ} 1, \mathrm{p} .27$.

Banque de données terminologique, $\mathrm{n}^{\circ} 1, \mathrm{p} .16$.

Banques de terminologies informatisées, $n^{\circ} 2$, p. 244.

Base de connaissances de la désamguïsation sémantique, $n^{\circ} 2$, p. 357.

Base de connaissances terminologiques (BCT), $\mathrm{n}^{\circ} 2$, p. 251.

Base de données terminographiques, $n^{\circ} 1$, p. 112.

Base de données terminologique multilingue (arabe, français, anglais), $\mathrm{n}^{\circ} 2, \mathrm{p} .321$.

Base terminologique, $n^{\circ} 1$, p. 20.

Base textuelle, $n^{\circ} 1$, p. 112 .

Base verbale, $n^{\circ} 1$, p. 18.

Basque actuel, $n^{\circ} 2$, p. 257.

Belleil, Claude et Denis Maurel : Un dictionnaire relationnel des noms propres liés à la géographie consulté par transducteurs, $\mathrm{n}^{\circ} 2$, p. 273.

Blanchon, Élisabeth: Point de vue sur la définition, $\mathrm{n}^{\circ} 1$, p. 168
Blanco, Xavier : Lexicographie bilingue (françaisespagnol) et traduction: l'exemple, $\mathrm{n}^{\circ} 1, \mathrm{p} .133$.

Bruneau, Cécile, Marina de Almeida, Margreet de Rooij, Marie-Hélène Lattaro, Mayra Parra, Justine Ravonihasindaza et Hélène Saulnier: Je construis ma maison. Étude lexico-terminologique de la construction, $\mathrm{n}^{\circ} 4$, p. 685 .

Clas, André et Gaston Gross : Synonymie, polysémie et classes d'objets, $\mathrm{n}^{\circ} 1, \mathrm{p} .147$.

Clas, André (sous la direction) : Lexicologie et terminologie $I I, \mathrm{n}^{\circ} 2$, p. 227.

Classe(s) d'objets, $\mathrm{n}^{\circ} 1$, p. $148 ; \mathrm{n}^{\circ} 1$, p. 156 ; $n^{\circ} 2$, p. 255.

Clavier, Viviane et Muriel Coret: $\boldsymbol{U}$ n dictionnaire électronique pour la reconnaissance des formes dérivées, $\mathrm{n}^{\circ} 2$, p. 307.

Code signé, $\pi^{\circ} 3$, p. 557.

COGNITERM, $\mathrm{n}^{\circ} 2$, p. 251

Collet, Tanja : La réduction des unités terminologiques complexes de type syntagmatique, $n^{\circ} 1$, p. 193.

Collocation, $n^{\circ} 1$, p. 15.

Collocation définitoire, $\mathrm{n}^{\circ} 1, \mathrm{p} .37$.

Combinaisons lexicales spécialisées, $\mathrm{n}^{\circ} 1$, p. 15.

Combinatoire, $\mathrm{n}^{\circ} 1$, p. 5.

Commerce de détail, $\mathrm{n}^{\circ} 2$, p. 234.

Communication non verbale, $n^{\circ} 3$, p. $488 ; n^{\circ} 3$, p. 497.

Compréhensionnisation, $\mathrm{n}^{\circ} 2$, p. 364 .

Concepts élémentaires d'un mot, $n^{\circ} 2$, p. 357.

Condamines, Anne et Josette Rebeyrolle : Point de vue en langue spécialisée, $\mathrm{n}^{\circ} 1, \mathrm{p} .174$.

Conditions d'énonciation, $\mathrm{n}^{\circ} 3$, p. 536 .

Construction du sens, $\mathrm{n}^{\circ} 1$, p. 95 .

Contente, Madalena et João Magalhães : Dictionnaire multilingue de médecine - vers une dictionnairique d'apprentissage, $\mathrm{n}^{\circ} 1$, p. 114.

Contexte-attestation, $\mathrm{n}^{\circ} 2$, p. 378.

Contexte définitoire, $n^{\circ} 2$, p. 378 .

Contexte explicatif, $n^{\circ} 2$, p. 378 .

Contexte illustratif, $n^{\circ} 2$, p. 378 .

Cooccurrent, $n^{\circ} 1$, p. 15.

Coordination, $n^{\circ} 1$, p. 193.

Cordón García, José Antonio : La traducción en España (1987-1993), n 4, p. 745.

Coret, Muriel et Viviane Clavier: Un dictionnaire électronique pour la reconnaissance des formes dérivées, $\mathrm{n}^{\circ} 2$, p. 307.

Corpus de textes économiques, $n^{\circ} 1$, p. 6 .

Création lexicale, $\mathrm{n}^{\circ} 1$, p. 102.

Dancette, Jeanne et Christophe Réthoré : Le dictionnaire bilingue (anglais-français) de la distribution : entre dictionnaire de langue et encyclopédie, $\mathrm{n}^{\circ} 2$, p. 229.

Déclivité culturelle, $\mathrm{n}^{\circ} 3$, p. 543 .

Décontextualisation, $n^{\circ} 2$, p. 268.

Découpage du réel, $n^{\circ} 2$, p. 365. 
Définition analytique, $\mathrm{n}^{\circ} 2$, p. 375 .

Définition descriptive, $\mathrm{n}^{\circ} 2$, p. 375 .

Définition encyclopédique, $n^{\circ} 2$, p. 375 .

Définition générique, $\mathrm{n}^{\circ} 1$, p. 170.

Définition lexicographique, $\mathrm{n}^{\circ} 1$, p. 170.

Définition linguistique, $\mathrm{n}^{\circ} 1$, p. 170 .

Définition métalinguistique, $\mathrm{n}^{\circ} 1, \mathrm{p} .170$.

Définition nominale, $\mathrm{n}^{\circ} 1$, p. 170.

Définition opératoire, $\mathrm{n}^{\circ} 2$, p. 375

Définition ostensive, $n^{\circ} 2$, p. 376 .

Définition par analyse, $\mathrm{n}^{\circ} 1$, p. 170.

Définition paraphrastique, $n^{\circ} 2$, p. 375 .

Définition par comparaison, $\mathrm{n}^{\circ} 2$, p. 376 .

Définition par compréhension, $n^{\circ} 1$, p. 170.

Définition par contenu, $\mathrm{n}^{\circ} 2$, p. 375 .

Définition par contraste, $n^{\circ} 2$, p. 376.

Définition par dénotation, $\mathrm{n}^{\circ} 2$, p. 375 .

Définition par implication, $n^{\circ} 2$, p. 375 .

Définition référentielle, $n^{\circ} 1$, p. 170 .

Définition(s), $n^{\circ} 1$, p. $36 ; n^{\circ} 1$, p. $168 ; n^{\circ} 2$, p. 374.

Définitions d'ouvrages spécialisés, ${ }^{\circ} 2$, p. 374 .

Delanghe, Olivier : La place de l'interprétation dans l'itinéraire scolaire, professionnel et universitaire d'un sourd, $\mathrm{n}^{\circ} 3, \mathrm{p} .555$.

Démarche d'un traducteur humain, $n^{\circ} 4$, p. 678.

Dénomination, $\mathrm{n}^{\circ} 1$, p. 194.

Dépistage automatique des termes, $\mathrm{n}^{\circ}$ 1, p. 207.

Dépouillement lexicographique, $\mathrm{n}^{\circ} 2$, p. 258 .

Dépouillement terminologique, $\mathrm{n}^{\circ} 1, \mathrm{p} .45$.

Dérivation, $\mathrm{n}^{\circ} 1$, p. 102.

Désambiguïsation, $\mathrm{n}^{\circ} 1$, p. 148

Désambiguïsation sémantique, $\mathrm{n}^{\circ} 2$, p. 356 .

Descripteur, $\mathrm{n}^{\circ} 1$, p. 207.

Descripteurs complexes, $\mathbf{n}^{\circ} 1$, p. 208

Diagrammaire, $n^{\circ} 3$, p. 494.

Dichy, Joseph : Pour une lexicomatique de l'arabe: l'unité lexicale simple et l'inventaire fini des spécificateurs du domaine du mot, ${ }^{\circ} 2$, p. 291.

Dictionnaire bilingue, $n^{\circ} 1$, p. $133 ; n^{\circ} 2$, p. 229.

Dictionnaire bilingue arabe / français, $n^{\circ} 1$, p. 142.

Dictionnaire contextuel du français économique, $\mathrm{n}^{\circ} 1$, p. 5.

Dictionnaire de morphèmes, $\mathrm{n}^{\circ} 1$, p. 72.

Dictionnaire, élaboration d'un, $\mathrm{n}^{\circ} 2$, p. 308 .

Dictionnaire électronique, $\mathrm{n}^{\circ} 1$, p. $72 ; \mathrm{n}^{\circ} 2$, p. 307.

Dictionnaire électronique relationnel, $\mathrm{n}^{\circ} 2$, p. 274 .

Dictionnaire français-malgache, $n^{\circ} 2$, p. 349 .

DIctionnaire INformatisé de l'ARabe, $n^{\circ} 2$, p. 291.

Dictionnaire interactif multilingue, $\mathrm{n}^{\circ} 1, \mathrm{p} .110$.

Dictionnaire, modèle d'organisation de, $\mathrm{n}^{\circ} 2, \mathrm{p} .308$.

Dictionnaire morphologique, $n^{\circ} 2$, p. 307 .

Dictionnaire multilingue informatisé de médecine, $\mathrm{n}^{\circ} 1, \mathrm{p} .115$.

Dictionnaires bilingues espagnol-français, $\mathrm{n}^{\circ} 1, \mathrm{p} .133$.

Dictionnaires bilingues français-espagnol, $\mathrm{n}^{\circ} 1$, p. 133 .

Dictionnaires bilingues généraux, $\mathrm{n}^{\circ} 1$, p. 55 .

Dictionnaires de spécialités, $\mathrm{n}^{\circ} 1$, p. 68

Dictionnaires, exploitation et l'édition de, $n^{\circ} 2$, p. 321 .

Dictionnaires informatiques, $n^{\circ} 2$, p. 283.
Dictionnaires multilingues africano-européens, $n^{\circ} 2$, p. 264

Dictionnaire spécialisé informatisé, $\mathrm{n}^{\circ} 1$, p. 115.

Dictionnaires terminologiques, $\mathrm{n}^{\circ} 1$, p. 24 .

Dictionnaire sur CD-ROM, $\mathrm{n}^{\circ} 1$, p. 25.

Dictionnaire virtuel, $\mathrm{n}^{\circ} 1$, p. 30 .

Dictionnairique, $\mathrm{n}^{\circ} 1$, p. 24.

Dictionnairique d'exploitation, $\mathrm{n}^{\circ} 1, \mathrm{p} .27$.

Diki-Kidiri, Marcel, Chérif Mbodj et Atibakwa Baboya Edema: Des lexiques en langues africaines (sängö, wolof, lingála) pour l'utilisateur de l'ordinateur, $\mathrm{n}^{\circ} 1$, p. 94.

Discours spécialisé, $\mathrm{n}^{\circ} 1$, p. 174.

Distribution, domaine de la, $\mathrm{n}^{\circ} 2$, p. 231.

Divergence de traduction, $\mathrm{n}^{\circ} 1, \mathrm{p} .135$.

Dorlian, Georges: Préalables linguistiques pour une traduction assistée par ordinateur du système verbal arabe-français, $\mathrm{n}^{\circ}$ 2, p. 317 .

Drouin, Patrick et Jacques Ladouceur : Une analyse terminométrique pour le repérage automatique des descripteurs complexes dans les textes de spécialité, $\mathrm{n}^{\circ} 1$, p. 207.

Drouin, Patrick : Une méthodologie d'identification automatique des syntagmes terminologiques: l'apport de la description du non-terme, $\mathrm{n}^{\circ} 1, \mathrm{p} .45$.

Durieux, Christine: La recherche terminologique en traduction: pour une approche hypertextuelle, $\mathrm{n}^{\circ} 4$, p. 677.

Edema, Atibakwa Baboya, Marcel Diki-Kidiri et Chérif Mbodj et : Des lexiques en langues africaines (sängö, wolof, lingála) pour l'utilisateur de l'ordinateur, $\mathrm{n}^{\circ}$ 1, p. 94.

Équivalence, $\mathrm{n}^{\circ} 2$, p. 365 .

Équivalence partielle, $n^{\circ} 2$, p. 365 .

Équivalence relative, $n^{\circ} 2$, p. 365 .

Équivalence totale, $\mathrm{n}^{\circ} 2$, p. 365 .

Équivalents non contextuels, $\mathrm{n}^{\circ} 1$, p. 143.

Équivalents non isomorphiques, $\mathrm{n}^{\circ} 1, \mathrm{p} .145$.

Équivalents périphrastiques, $\mathrm{n}^{\circ} 1$, p. 143 .

EUSKALTERM (Centre Basque de Terminologie), $n^{\circ} 2$, p. 257.

Exemple, $\mathrm{n}^{\circ} 1$, p. 133.

Exploitation de textes, $n^{\circ} 2$, p. 321 .

Expressions figées, $n^{\circ} 2$, p. 348

Extensionnisation, $\mathrm{n}^{\circ} 2$, p. 364 .

Extraction automatique de vocabulaire spécialisé, $n^{\circ} 2$, p. 328.

Extraction de termes, $\mathbf{n}^{\circ} 2$, p. 321 .

Fausse transparence, $n^{\circ} 3$, p. 542 .

Fiche terminologique, $n^{\circ} 2$, p. $328 ; n^{\circ} 4$, p. 686 .

Filtrage, $n^{\circ} 1$, p. 48.

Filtrage des candidats, $\mathrm{n}^{\circ} 1$, p. 210

Filtrage des non-termes, $\mathrm{n}^{\circ}$ 1, p. 210.

Filtrage par analyse contextuelle, $\mathrm{n}^{\circ}$ 1, p. 212.

Fonction syntaxique, $\mathbf{n}^{\circ} 2$, p. 357 .

Formalisme à deux niveaux, $\mathrm{n}^{\circ} 1, \mathrm{p} .73$. 
Fournier, Christiane : L'interprétation pour sourds au pénal en France, $\mathrm{n}^{\circ}$ 3, p. 533.

Fraïssé, Sylvain et Eugène Sandford: Outil d'extraction de la sémantique d'un corpus textuel, $\mathrm{n}^{\circ} 2$, p. 356 .

Français économique, $\mathrm{n}^{\circ} 1$, p. 5.

Français signé, $\mathrm{n}^{\circ} 3$, p. 557.

FRANTEXT, $\mathbf{n}^{\circ}$ 2, p. 253.

Garcia, Maria de Lurdes Abrantes: Projet de dictionnaire interactif multilingue de termes médicaux, $\mathrm{n}^{\circ} 1$, p. 110.

Génération automatique d'éléments morphologiques, $\mathrm{n}^{\circ} 2$, p. 321 .

Gesché, Véronique : Évaluation des définitions d'ouvrages, $n^{\circ} 2$, p. 374.

Gestes descriptifs, $n^{\circ} 3$, p. 497

Gestualité conversationnelle, $\mathrm{n}^{\circ} 3$, p. 491.

Gouadec, Daniel : Dictionnaires terminologiques L'impact des nouvelles technologies, $\mathrm{n}^{\circ} 1, \mathrm{p} .24$.

Grammaire syntagmatique guidée par les têtes, $n^{\circ} 1$, p. 72 .

Graphies multiples, $n^{\circ} 2$, p. 275.

Gréciano, Gertrud : Collocations rythmologiques, $\mathrm{n}^{\circ} 1, \mathrm{p} .33$.

Gross, Gaston et André Clas : Synonymie, polysémie et classes d'objets, $\mathrm{n}^{\circ} 1, \mathrm{p} .147$.

Histoire de la traduction aux Pays-Bas, $\mathrm{n}^{\circ} 4$, p. 741. Hlal, Yahya : Outils linguistiques et système terminologique multilingue, $\mathrm{n}^{\circ} 2$, p. 321 .

Homonymie, $n^{\circ} 1$, p. 174

Hypertexte, $n^{\circ} 4$, p. 680 .

Identification automatique des descripteurs, $\mathrm{n}^{\circ} 1, \mathrm{p} .208$.

Idiomes, $\mathrm{n}^{\circ} 2$, p. 347 .

Igbo, $\mathrm{n}^{\circ} 4$, p. 642

Images langagières, $n^{\circ} 1$, p. 39

Index, $\mathbf{n}^{\circ} 2$, p. 328 .

Information terminographique, $\mathrm{n}^{\circ} 1, \mathrm{p} .16$.

Insertion, $n^{\circ} 1$, p. 194.

Instrumentation des langues africaines, $n^{\circ} 2$, p. 264. Internet, $\mathrm{n}^{\circ} 2$, p. 283.

Interprétation au pénal, $n^{\circ} 3$, p. 534.

Interprétation de conférence en LSF, $n^{\circ} 3$, p. 521 ; $\mathrm{n}^{\circ} 3$, p. 529

Interprétation de français en LSF, $\mathrm{n}^{\circ} 3, \mathrm{p} .529$. Interprétation de $\mathrm{LSF}$ en français, $n^{\circ} 3$, p. 528 .

Interprétation en langues des signes, $n^{\circ} 3$, p. 485.

Interprétation pour les personnes sourdes, $n^{\circ} 3$, p. 533

Labialisation, $n^{\circ} 3$, p. 488

Ladouceur, Jacques et Patrick Drouin: Une analyse terminométrique pour le repérage automatique des descripteurs complexes dans les textes de spécialité, $\mathrm{n}^{\circ} 1, \mathrm{p} .207$

Langue des signes, $n^{\circ} 3$, p. 485.
Langue française des signes (LSF), $\mathrm{n}^{\circ} 3$, p. 485.

Langues spécialisées, $n^{\circ}$ 1, p. 174.

Lattaro, Marie-Hélène, Mayra Parra, Justine Ravonihasindaza, Hélène Saulnier, Cécile Bruneau, Marina de Almeida et Margreet de Rooij : Je construis ma maison. Étude lexicoterminologique de la construction, $\mathrm{n}^{\circ} 4, \mathrm{p} .685$.

Lecteur d'index, $n^{\circ} 2$, p. 328.

Lecture labiale, $\mathrm{n}^{\circ} 3$, p. $489 ; \mathrm{n}^{\circ} 3$, p. 556.

Lemmatisation, $n^{\circ} 2$, p. 257.

Lepage, Thierry et Caroline de Schaetzen : Evaluation $d^{\prime}$ un échantillon d'index, $\mathrm{n}^{\circ} 2, \mathrm{p} .328$.

Lexicographie congolaise, $n^{\circ} 2$, p. 264.

Lexicomatique, $n^{\circ} 2$, p. 364 .

Lexicomatique de l'arabe, $\mathrm{n}^{\circ}$ 2, p. 291.

Lexique basque, $\mathrm{n}^{\circ} 2$, p. 257.

L'Homme, Marie-Claude: Méthode d'accès informatisé aux combinaisons lexicales en langue technique, $\mathrm{n}^{\circ} 1$, p. 15.

Lingála, $\mathrm{n}^{\circ} 1$, p. 95 .

Lipou, Antoine : Dictionnaires multilingues généraux en langues africaines, $\mathrm{n}^{\circ} 2$, p. 264.

Localisations spatiales, $\mathrm{n}^{\circ} 3$, p. 498.

Locution nominale de spécialité, $\mathrm{n}^{\circ} 1$, p. 68.

LSF, $n^{\circ} 3$, p. 485 .

Lyadri, Rachid: Problématique des équivalences sémantiques et de la traduction dans des dictionnaires arabe-français, $\mathrm{n}^{\circ}$ 1, p. 142.

Magalhães, João et Madalena Contente : Dictionnaire multilingue de médecine - vers une dictionnairique d'apprentissage, $\mathrm{n}^{\circ} 1$, p. 114.

Malentendant, $n^{\circ} 3$, p. 540.

Malgache, $n^{\circ}$ 2, p. 347 .

Marché dictionnairique, $\mathrm{n}^{\circ} 1$, p. 29.

Matra Marconi Space, $n^{\circ}$ 2, p. 252.

Maurel, Denis et Claude Belleil : Un dictionnaire relationnel des noms propres liés à la géographie consulté par transducteurs, $\mathrm{n}^{\circ}$ 2, p. 273.

Mbodj, Chérif, Atibakwa Baboya Edema et Marcel Diki-Kidiri : Des lexiques en langues africaines (sängö, wolof, lingála) pour l'utilisateur de l'ordinateur, $\mathrm{n}^{\circ} 1$, p. 94.

Médiostructure du dictionnaire, $\mathrm{n}^{\circ}$ 2, p. 237.

Métaphores, $n^{\circ}$ 4, p. 643.

Méthodologie de repérage de polyacceptions, $\mathrm{n}^{\circ} 1$, p. 174

Meunier-Crespo, Mariette : Les locutions nominales dans les dictionnaires de spécialités, $\mathrm{n}^{\circ} 1, \mathrm{p} .68$.

Microstructure du dictionnaire, $n^{\circ} 2$, p. 235.

Micro-syntaxe, $\mathrm{n}^{\circ} 1$, p. 69.

Mimes, $n^{\circ} 3$, p. 497.

Mimiques, $n^{\circ} 3$, p. 497

Mondialisation, $\mathrm{n}^{\circ} 1$, p. 27

Morphologie à deux niveaux, $\mathrm{n}^{\circ} 1, \mathrm{p} .72$.

Morphologie dérivationnelle, ${ }^{\circ} 2$, p. 276

Morphologie flexionnelle, $\mathbf{n}^{\circ} 2$, p. 275.

Mots dérivés, description des, $\mathrm{n}^{\circ} 2$, p. 308 .

Mots suffixés du français, $n^{\circ} 2$, p. 307.

M-spécificateurs, $n^{\circ} 2$, p. 301.

Muet, $n^{\circ} 3$, p. 540. 
Namer, Fiammetta et Paul Schmidt: Construction d'un dictionnaire : morphologie à deux niveaux pour le français à l'aide de contraintes basées sur les structures de traits typées, $n^{\circ} 1, p .72$.

Néo-dictionnairique, $\mathrm{n}^{\circ} 1, \mathrm{p} .30$.

Néologie raisonnée, $\mathrm{n}^{\circ} 1$, p. 111

Néologisme, aide au, $n^{\circ} 2$, p. 321 .

Néonymes, $\mathrm{n}^{\circ} 1$, p. 111 .

Niveaux de langue, $\mathrm{n}^{\circ} 1$, p. 55

Nom propre, $n^{\circ} 2$, p. 273

Non-attendu, notion de, $n^{\circ} 2$, p. 273

Non-standard, $\mathbf{n}^{\circ} 1$, p. 56.

Non-termes, $\mathrm{n}^{\circ} 1$, p. 211

Normalisation, $\mathbf{n}^{\circ} 1$, p. $28 ; \mathrm{n}^{\circ} 2$, p. 257.

Notions, $n^{\circ} 1$, p. 212 .

Opérateur, $\mathrm{n}^{\circ} 1$, p. 147

Opérateurs verbaux, $\mathrm{n}^{\circ} 2$, p. 255.

Orthosignes, $\mathrm{n}^{\circ} 3$, p. 488.

Otman, Gabriel : Les bases de connaissances terminologiques: les banques de terminologie de seconde génération, $\mathrm{n}^{\circ} 2$, p. 244.

Outil de consultation de données lexicales, $n^{\circ} 2$, p. 283

Outils dictionnairiques, $\mathrm{n}^{\circ} 1$, p. 25 .

Pak, Man-ghyu : Traduction automatique et classes d'objets : le problème de porter un vêtement en français et en coréen, $\mathrm{n}^{\circ} 1, \mathrm{p} .155$.

Paradigme d'une science, $\mathrm{n}^{\circ}$ 1, p. 114.

Parra, Mayra, Justine Ravonihasindaza, Hélène Saulnier, Cécile Bruneau, Marina de Almeida Margreet deRooij et Marie-Hélène Lattaro : Je construis ma maison. Étude lexico-terminologique de la construction, $n^{\circ} 4$, p. 685.

Paulin, Aurélia: Analyse de la notion de non-standard dans les dictionnaires bilingues français-anglais, $n^{\circ} 1$, p. 55.

Pédagogie strictement oraliste, $n^{\circ} 3$, p. 561 .

Petitpierre, Dominique et Gilbert Robert : DICO : un outil de consultation de dictionnaaire en réseau. $\mathrm{n}^{\circ} 2$, p. 283

Phraséologie, $n^{\circ} 1$, p. 29.

Phraséologie scientifique, $n^{\circ} 1$, p. 33.

Phraséologisme, $n^{\circ} 1$, p. 15.

Phraséotermes, $n^{\circ} 1$, p. 34

Polyacception, $n^{\circ} 1$, p. 174

Polylexicalité terminologique, $\mathrm{n}^{\circ} 1, \mathrm{p} .33$.

Polysémie, $\mathrm{n}^{\circ} 1$, p. $155 ; \mathrm{n}^{\circ} 1$, p. 174.

Prédication, $\mathrm{n}^{\circ} 1$, p. 194.

Prédicats nominaux, $\mathrm{n}^{\circ} 1, \mathrm{p} .148$

Prédicats verbaux, $\mathrm{n}^{\circ} 1, \mathrm{p} .148$.

Prise en note simultanée, $n^{\circ} 3$, p. 524.

Programme client, $n^{\circ} 2$, p. 284.

Quasilinguistiques, $n^{\circ}$ 3, p. 497.
Raharinirina Rabaovololona, Lucie : Problèmes posés par les expressions ou idiomes dans la construction d'un dictionnaire d'usage bilingue, $\mathrm{n}^{\circ} 2$, p. 347

Ravonihasindaza, Justine, Hélène Saulnier, Cécile Bruneau, Marina de Almeida, Margreet de Rooij, Marie-Hélène Lattaro et Mayra Parra : Je construis ma maison. Étude lexico-terminologique de la construction, $\mathrm{n}^{\circ} 4$, p. 685 .

Rebeyrolle, Josette et Anne Condamines: Point de vue en langue spécialisée, $\mathrm{n}^{\circ} 1, \mathrm{p} .174$.

Recherche documentaire ${ }^{\circ} 4$, p. 686.

Recherche terminologique, $\mathrm{n}^{\circ} 4$, p. 677

Réduction, $\mathbf{n}^{\circ}$ 1, p. 195

Réduction à caractère lexical, $\mathrm{n}^{\circ} 1$, p. 198.

Relations lexico-sémantiques, $n^{\circ} 2$, p. 230.

Relations sémantiques, $\mathrm{n}^{\circ} 2$, p. 245.

Relations syntaxiques, $\mathrm{n}^{\circ} 1$, p. 161.

Repérage de la terminologie, $\mathrm{n}^{\circ} 1$, p. 207.

Repérage des candidats-termes, $\mathrm{n}^{\circ} 1$, p. 48 .

Repérage de termes, $n^{\circ} 1$, p. 45

Reprise anaphorique, $\mathrm{n}^{\circ} 1$, p. 198.

Réseau sémantico-terminologique (RST), $\mathrm{n}^{\circ} 2$, p. 245.

Réseaux internotionnels, $\mathrm{n}^{\circ} 2$, p. 234

Réthoré, Christophe et Jeanne Dancette : $L e$ dictionnaire bilingue (anglais-français) de la distribution : entre dictionnaire de langue et encyclopédie, $\mathrm{n}^{\circ} 2$, p. 229.

Robert, Gilbert et Dominique Petitpierre : DICO : un outil de consultation de dictionnaire en réseau, $n^{\circ} 2$, p. 283.

Rôles sémantiques, $\mathrm{n}^{\circ} 1$, p. 38 .

Rooij, Margreet de, Marie-Hélène Lattaro, Mayra Parra, Justine Ravonihasindaza, Hélène Saulnier, Cécile Bruneau et Marina de Almeida : Je construis ma maison. Étude lexico-terminologique de la construction, $\mathrm{n}^{\circ} 4$, p. 685 .

Sandford, Eugène et Sylvain Fraïssé : Outil d'extraction de la sémantique d'un corpus textuel, $\mathrm{n}^{\circ} 2, \mathrm{p} .356$.

Sängö, $n^{\circ} 1$, p. 95 .

Saulnier,Hélène, Cécile Bruneau, Marina de Almeida, Margreet de Rooij, Marie-Hélène Lattaro, Mayra Parra et Justine Ravonihasindaza : Je construis ma maison. Étude lexico-terminologique de la construction, $\mathrm{n}^{\circ} 4$, p. 685 .

Schaetzen, Caroline de et Thierry Lepage : Évaluation $d^{\prime} u n$ échantillon d'index, $\mathrm{n}^{\circ} 2$, p. 328 .

Schmidt, Paul et Fiammetta Namer : Construction d'un dictionnaire : morphologie à deux niveaux pour le francais à l'aide de contraintes basées sur les structures de traits typées, $\mathrm{n}^{\circ} 1, \mathrm{p} .72$.

Seleskovitch, Danica : Interview de Mme Arlette Morel, présidente de la fédération nationale des sourds de France, $\mathrm{n}^{\circ} 3$, p. 560 .

Seleskovitch, Danica (sous la direction) : L'interprétation en langues des signes, $\mathrm{n}^{\circ} 3, \mathrm{p} .485$.

Sénologie, $\mathrm{n}^{\circ} 1$, p. 110. 
Sens d'un mot, $n^{\circ} 2$, p. 357.

Séquence $L 1, \mathrm{n}^{\circ} 1, \mathrm{p} .133$.

Séquence L2, $\mathrm{n}^{\circ} 1$, p. 133.

Séro-Guillaume, Philippe : La langue des signes française (LSF), no 3, p. 485.

Séro-Guillaume, Philippe : Pourquoi une formation à l'interprétation de conférence LSF (langue des signes française) en français et de français en $\operatorname{LSF}$ ? $\mathrm{n}^{\circ} 3, \mathrm{p} .521$.

Serveur de dictionnaires, $n^{\circ} 2$, p. 284.

Serveur de données, $\mathrm{n}^{\circ} 2$, p. 285

Serveur d'informations, $\mathrm{n}^{\circ} 2$, p. 284.

Signes manuels, $\mathrm{n}^{\circ} 3$, p. 495.

Signifiants de caractère imitatif, $n^{\circ} 3$, p. 491.

Signifiants labiaux, $n^{\circ} 3$, p. 488 .

Socioterminologie dans le dictionnaire, $\mathrm{n}^{\circ} 2$, p. 239.

Sourdisme, $\mathrm{n}^{\circ} 3$, p. 521 .

Sourd-muet, $n^{\circ} 3$, p. 540

Sourd profond, $\mathrm{n}^{\circ} 3$, p. 555.

Sous-catégorisation morphologique, $\mathrm{n}^{\circ}$ 1, p. 73.

Spécificateurs, $n^{\circ} 2$, p. 291.

Spécificateurs morphosyntaxiques du domaine du mot, $\mathbf{n}^{\circ} 2$, p. 291.

Standardisation, $\mathrm{n}^{\circ} 1$, p. 28.

St-Pierre, Paul (sous la direction) : Traduction et post-colonialisme en Inde, $\mathrm{n}^{\circ} 2$, p. 391.

Stratégies de l'interprétation, $n^{\circ} 3$, p. 537.

Stratégies lexicographiques, $\mathrm{n}^{\circ} 2$, p. 229.

Structuration des connaissances, $\mathrm{n}^{\circ} 2$, p. 234

Structure de la définition, $\mathrm{n}^{\circ} 1, \mathrm{p} .168$.

Structure morphologique des mots dérivés par suffixation, $n^{\circ} 2$, p. 307

Structures de traits typées, $n^{\circ} 1$, p. 72 .

Stylistique, $\mathrm{n}^{\circ} 4$, p. 642 .

Syntagme terminologique, $n^{\circ} 1$, p. 193.

Synthèse et analyse morphosyntaxiques informatisées de l'arabe, $\mathrm{n}^{\circ} 2$, p. 291.

Système notionnel, n ${ }^{\circ}$, p. 245.

Termes, $\mathrm{n}^{\circ} 1$, p. 207.

Terme(s) complexe(s), no 1, p. $208 ; n^{\circ} 1$, p. 209

Termes monolexicaux, $n^{\circ} 1$, p. 34

Termes polylexicaux, $n^{\circ} 1$, p. 34 .

Termes-vedettes, $n^{\circ} 1, p .33$.

Terminodidactiquc, $\mathrm{n}^{\circ} 1$, p. 111.

Terminologie, $n^{\circ} 4$, p. 677 .

Terminologie bilingue, $n^{\circ} 2$, p. 229 .
Terminologie de géomorphologie, $\mathrm{n}^{\circ} 2$, p. 246

Terminologie de la sénologie, $\mathrm{n}^{\circ} 1$, p. 111.

Tête, $\mathrm{n}^{\circ}$ 1, p. 73.

Tondji-Simen, René : Lexicomatique, compréhensionnisation et extensionnisation, $\mathrm{n}^{\circ} 2$, p. 364 .

Topicalisation, $\mathrm{n}^{\circ} 3$, p. 498.

Traduction automatique, $\mathrm{n}^{\circ} 1, \mathrm{p} .155$.

Traduction de l'exemple, $n^{\circ} 1$, p. 133.

Traduction des textes littéraires, $n^{\circ} 4$, p. 641 .

Traduction français / arabe, $\mathrm{n}^{\circ} 1$, p. 142.

Traduction hétéromorphe, $\mathbf{n}^{\circ} 1$, p. 135.

Traduction littéraire, $\mathrm{n}^{\circ} 4$, p. 642.

Traductions isomorphes, $\mathrm{n}^{\circ} 1$, p. 134

Traitement automatique, $n^{\circ} 1$, p. 148.

Traitement automatique de la langue naturelle, $\mathrm{n}^{\circ} 2$, p. 356 .

Traitement automatique des noms propres, $n^{\circ} 2$, p. 273.

Traitement automatique du langage, $\mathrm{n}^{\circ} 1, \mathrm{p} .72$.

Traitement de la polysémie, ${ }^{\circ} 1$, p. 155.

Traits syntactico-sémantiques, $\mathrm{n}^{\circ} 1$, p. 148.

Traits syntaxiques, $\mathrm{n}^{\circ} 1, \mathrm{p} .148 ; \mathrm{n}^{\circ} \mathrm{l}, \mathrm{p} .155$.

Transcription phonétique, $\mathrm{n}^{\circ} 2, \mathrm{p} .266$.

Transducteur de hachage, $n^{\circ} 2$, p. 277.

Types de noms propres, $\mathrm{n}^{\circ} 2$, p. 278.

Unité lexicale complexe, $\mathrm{n}^{\circ} 1$, p. 209

Unité lexicale simple, $n^{\circ} 2$, p. 291.

Urkia, Miriam et Jose Antonio Aduriz: La lexicographie assitée par ordinateur. L'expérience d'Uzei, $\mathrm{n}^{\circ} 2$, p. 257

UZEI (Centre basque pour la normalisation linguistique), $\mathrm{n}^{\circ} 2$, p. 257.

Van Hoof, Henri : Le XVIII ${ }^{e}$ siècle néerlandais : un méconnu pour la traduction, $n^{\circ} 4$, p. 741.

Variantes topolectales, $n^{\circ} 2$, p. 369

Vecteurs pour les phrases et les textes, $n^{\circ} 2$, p. 357.

Verlinde, Serge : Le vocabulaire des fluctuations dans le discours économique : synonymie et combinatoire, $n^{\circ} 1$, p. 5 .

VITASOA, n ${ }^{\circ}$ 2, p. 349.

Vocabulaire des fluctuations, $n^{\circ} 1$, p. 5 .

Wolof, $n^{\circ} 1$, p. 95. 


\section{Index anglais des mots et des sujets traités}

Adaptation/translation, $\mathrm{n}^{\circ} 2$, p. 417 .

Alignment, $n^{\circ} 4$, p. 608 .

American Sign Language (ASL), n ${ }^{\circ} 3$, p. 485; $\mathrm{n}^{\circ} 3$, p. 502.

Anglo-centrism, $n^{\circ} 2$, p. 417 .

Annotation standards, $n^{\circ} 4$, p. 607 .

Anti-colonial nationalism, $n^{\circ} 2$, p. 444.

Appropriation, $\mathrm{n}^{\circ} 2$, p. 416 .

Arabic-English translation, $n^{\circ} 4$, p. 671 .

Arabic jokes, $n^{\circ} 4$, p. 671 .

Archibald, James: The Pragmatics of Profes-

sionalism: Translation and Interpretation in

Puerto Rico and Quebec, $\mathrm{n}^{\circ} 4$, p. 649.

ASL, $n^{\circ} 3$, p. 515.

ASL and English, n ${ }^{\circ} 3$, p. 515.

ASL / English interpreters, $n^{\circ} 3$, p. 517.

ASL grammar, $n^{\circ} 3$, p. 503 .

Bilingual dictionary, $\mathrm{n}^{\circ} 1$, p. 121 .

British colonial rule, $n^{\circ} 2$, p. 424 .

Cao, Deborah: Consideration in Translating

English / Chinese Contracts, $n^{\circ} 4$, p. 661

Centre for German Sign Language and

Communication of the Deaf, $\mathrm{n}^{\circ} 3, \mathrm{p} .548$.

Charles Dickens's A Tale of Two Cities, $\mathbf{n}^{\circ} 2$, p. 417.

Charter of the French language, $n^{\circ} 4$, p. 656 .

Chinese legal system, $n^{\circ} 4$, p. 662 .

Choudhuri, Indra Nath: The Plurality of Languages and Literature in Translation: The Post-colonial Context, $\mathrm{n}^{\circ} 2$, p. 439

Colonization, effects of, $n^{\circ} 2, p .424$

Conjunctives, $n^{\circ} 4$, p. 630 .

Consecutive interpreting (CI), $\mathrm{n}^{\circ} 4$, p. 616 .

Context, $n^{\circ} 1$, p. 128.

Corpora Annotation, $\mathrm{n}^{\circ} 4$, p. 607.

Corpus-based linguistics, $n^{\circ} 4$, p. 606 .

Corpus-based MT, ${ }^{\circ} 4$, p. 606.

Corpus computational linguistics, $\mathrm{n}^{\circ} 4$, p. 606 .

Cross cultural communication, $\mathrm{n}^{\circ} 3$, p. 516.

Cultural identity, $n^{\circ} 2$, p. 463 .

Cultural pluralism, positive value of, $n^{\circ} 2$, p. 439 .
Cultural references, $n^{\circ} 2$, p. 424 .

Cultural studies, $\mathbf{n}^{\circ} 2$, p. 462 .

Cultural turn in translation, $n^{\circ} 2, p .464$

Culture, boundaries of, $n^{\circ} 2, p .463$

Darò, Valeria: Experimental Studies on Memory in Conference Interpretation, $n^{\circ} 4$, p. 622 .

Deaf community in Germany, $n^{\circ} 3$, p. 546.

Deaf signers, $\mathrm{n}^{\circ} 3$, p. 503 .

Déjean Le Féal, Karla: Simultaneous Interpretation with "Training wheels", n०4, p. 616

Devy, G. N.: Literary History and Translation: An Indian View, $\mathrm{n}^{\circ} 2$, p. 395.

Distance calculation, $\mathrm{n}^{\circ} 4$, p. 610 .

Editing protocol, $\mathrm{n}^{\circ} 1$, p. 122 ,

El-Yasin, Mohammad K.: The Translatability of Arabic Jokes into English, ${ }^{\circ} 4$, p. 670.

English / Chinese legal documents, $n^{\circ} 4$, p. 661 .

English / Chinese translation, $n^{\circ} 4$, p. 661 .

English literature, supremacy of, $n^{\circ} 2$, p. 408.

Episodic memory, $n^{\circ} 4$, p. 624 .

Example-based MT, $\mathrm{n}^{\circ} 4$, p. 605 .

Expert Advisory Group on Language Engineering Standards (EAGLES), n ${ }^{\circ} 4$, p. 607

Explicit memory, $n^{\circ} 4$, p. 623.

Extra-literary charge, $n^{\circ} 2$, p. 408 .

Fakir Mohan Senapati, Chha Mana Atha Guntha, $n^{\circ} 2$, p. 424.

Farghal, Mohammed and Abdullah Shakir: When the Focus of the Text is Blurred:

A Textlinguistic Approach for Analyzing Student Interpreters' Errors, $n^{\circ} 4$, p. 629

Feminist translator, $n^{\circ} 2, p .470$.

French literature in Hindi, reception of, $n^{\circ} 2$, p. 413 .

French literature, superiority of, $n^{\circ} 2$, p. 412 .

Gathasaptashati, $\mathrm{n}^{\circ} 2$, p. 456 .

German Sign Language, $n^{\circ} 3$, p. 548 .

Grammar of ASL, $n^{\circ} 3$, p. 502 .

Hierarchization of the information, $\mathrm{n}^{\circ} 1, \mathrm{p} .127$.

Humphrey, Janice H.: Chopping Down and

Reconstructing a Tree, $n^{\circ} 3$, p. 515.

Hungarian-French dictionary, $\mathbf{n}^{\circ} 1$, p. 121.

Implicit memory, $n^{\circ} 4$, p. 623 .

Indian-English literature, $\mathrm{n}^{\circ} 2$, p. 402.

Indian epistemology, $\mathrm{n}^{\circ} 2$, p. 398.

Indian languages, $n^{\circ} 2$, p. 395 .

Indian languages, use of, $n^{\circ} 2$, p. 445

Indian literary scene, $n^{\circ} 2$, p. 408. 
Indian literary tradition, $\mathrm{n}^{\circ} 2$, p. 395 .

Indian literature, $\mathrm{n}^{\circ} 2$, p. 439

Indian sensibility, ${ }^{\circ} 2$, p. 409

Indian society, $\mathrm{n}^{\circ} 2$, p. 398.

Indian story, $\mathrm{n}^{\circ} 2, \mathrm{p} .452$.

Indian traditions, $\mathrm{n}^{\circ} 2$, p. 398.

Indian values, $\mathrm{n}^{\circ} 2$, p. 409

India(s), $n^{\circ} 2$, p. $393 ; n^{\circ} 2$, p. 451.

India's thinking, $n^{\circ} 2, \mathrm{p} .439$.

Intercultural negotiation, $\mathrm{n}^{\circ} 2$, p. 424.

Interpretation, $\mathrm{n}^{\circ} 3$, p. $503 ; \mathrm{n}^{\circ} 4$, p. 622 .

Interpreters of sign language, $n^{\circ} 3$, p. 547.

Janzen, Terry: Pragmatic and Syntactic Features of Topics in American Sign Language, $\mathrm{n}^{\circ} 3$, p. 502. Joke, ${ }^{\circ} 4$, p. 670 .

Kamala, N. and G. J. V. Prasad: Trans-creating India(s): The Nation in English Translation, $\mathrm{n}^{\circ} 2$, p. 450

Languages, power-centred, $n^{\circ} 2, p .451$.

Legal language, $n^{\circ} 4$, p. 662.

Legal tradition, $n^{\circ} 4$, p. 662.

Legal translation, $n^{\circ} 4$, p. 661.

Lexical cohesion, $\mathrm{n}^{\circ} 1$, p. 185.

Lexical collocation, $\mathrm{n}^{\circ} 1, \mathrm{p} .185$

Lexical items, $n^{\circ} 4$, p. 630 .

Lexicographical standards, $\mathrm{n}^{\circ} 1, \mathrm{p} .127$.

Linguistic identifications, $n^{\circ} 2$, p. 450

Literary translation(s), $\mathrm{n}^{\circ} 2$, p. $395 ; \mathrm{n}^{\circ} 2$, p. 402 $\mathrm{n}^{\circ} 2$, p. 456

Long term memory, $n^{\circ} 4$, p. 623.

Lotfipour-Saedi, K.: Lexical Cohesion and Translation Equivalence, $\mathrm{n}^{\circ}$ 1, p. 185.

Machine Translation (MT), $\mathrm{n}^{\circ} 4, \mathrm{p} .605$

Manual / visual representation, $\mathrm{n}^{\circ} 3$, p. 516 .

Martin de Santa Olalla Sánchez, Aurora and Celia Rico Pérez: New Trends in Machine Translation, $n^{\circ} 4$, p. 605

Memory in interpretation, $n^{\circ} 4$, p. 622

Mohapatra, Himansu S. and Jatindra K. Nayak: Translating against the grain: The Case of an Oriya Adaptation of Charles Dickens's A Tale of Two Cities, $n^{\circ} 2$, p. 416.

Mukherjee, Sujit: The Absent Traveller is Still Far From the River, $\mathrm{n}^{\circ} 2$, p. 456

Multilingualism, $\mathrm{n}^{\circ}$ 2, p. 439.

Multilingualism of India, $\mathrm{n}^{\circ} 2$, p. 440.

Nayak, Jatindra K. and Himansu S. Mohapatra: Translating against the grain: The Case of an Oriya Adaptation of Charles Dickens's A Tale of Two Cities, $\mathrm{n}^{\circ} 2$, p. 416.

Network of European Reference Corpora (NERC), $\mathrm{n}^{\circ} 4$, p. 605 .
Nilsson, Anna-Lena: Sign Language Interpreting in Sweden, $\mathrm{n}^{\circ} 3$, p. 550 .

Official language issue, $\mathrm{n}^{\circ} 4$, p. 650 .

Official language legislation, $n^{\circ} 4$, p. 656 .

Para-literature, $n^{\circ} 2$, p. 396

Parallel corpora, $\mathrm{n}^{\circ} 4$, p. 606.

Post-colonial, $\mathrm{n}^{\circ} 2$, p. 444

Postcolonial context, $n^{\circ} 2$, p. 424.

Post-colonial pedagogy, $n^{\circ} 2$, p. 471

Power, translation and, $\mathrm{n}^{\circ} 2$, p. 393.

Puerto Rico, $n^{\circ} 4$, p. 649.

Primary and secondary memory, $n^{\circ} 4$, p. 623.

Quebec, $n^{\circ} 4$, p. 656

Ramakrishna, Shantha: Functions of Translation in Post-Colonial India, $\mathrm{n}^{\circ} 2$, p. 444.

Rewriting, $n^{\circ} 2$, p. 417

Rico Pérez, Celia and Aurora Martin de Santa Olalla Sánchez: New Trends in Machine Translation, $\mathrm{n}^{\circ} 4$, p. 605 .

Schulz, Andrea: Sign Language Interpreting in Germany on the Way Towards Professionalism, $\mathrm{n}^{\circ} 3, \mathrm{p} .546$

Semantic memory, ${ }^{\circ} 4$, p. 624 .

Shadowing, $\mathrm{n}^{\circ} 4$, p. 617.

Shakir, Abdullah and Mohammed Farghal: When the Focus of the Text is Blurred: A Textlinguistic Approach for Analyzing Student Interpreters' Errors, $n^{\circ} 4$, p. 629.

Short term memory, $n^{\circ} 4$, p. 623 .

Signed languages, $n^{\circ} 3$, p. 502

Sign language interpreting, $n^{\circ} 3$, p. 546.

Simon, Sherry: Translation, Postcolonialism and Cultural Studies, $n^{\circ} 2$, p. 462

Simultaneous interpretation (SI), $\mathrm{n}^{\circ} 4$, p. 616

Simultaneous interpreting, $n^{\circ} 4$, p. 630 .

Standard Generalised Mark-up Language (SGML) $n^{\circ} 4$, p. 607.

Statistics-based MT, ${ }^{\circ} 4$, p. 605

St-Pierre, Paul: Translating Cultural Difference: Fakir Mohan Senapati's Chha Mana Atha Guntha, $n^{\circ} 2$, p. 423.

St-Pierre, Paul: Translation and Postcolonialism: India, $\mathrm{n}^{\circ} 2$, p. 391

Student interpreters, $n^{\circ} 4$, p. 631.

Swedish Sign Language, ${ }^{\circ} 3$, p. 551.

Syntax of ASL, $n^{\circ} 3$, p. 503.

Szende, Thomas: An Informal Report on the New Hungarian-French Dictionary, ${ }^{\circ} 1$, p. 121. 
Text Encoding Initiative (TEI), $\mathrm{n}^{\circ} 4$, p. 607.

Texture of a text, $\mathbf{n}^{\circ} 1$, p. 185 .

Three-dimentional space, $\mathrm{n}^{\circ} 3$, p. 503.

Topicality, n 3, p. 503.

Topic-comment structure in ASL, $\mathrm{n}^{\circ}$ 3, p. 503.

Translatability, $n^{\circ} 2$, p. 427.

Translatability of jokes, $n^{\circ} 4$, p. 672 .

Translating contracts, $\mathrm{n}^{\circ} 4$, p. 663 .

Translational culture, $\mathrm{n}^{\circ} 2$, p. 462 .

Translation and adaptation, $\mathrm{n}^{\circ} 2$, p. 417.

Translation equivalence, $n^{\circ} 1, p .185$

Translation, incompleteness of, $n^{\circ} 2$, p. 475

Translation in Indian culture, $n^{\circ} 2$, p. 395.

Translation in post-colonial India, functions of, $\mathrm{n}^{\circ} 2$, p. 444.

Translation of jokes, $n^{\circ} 4$, p. 671

Translation practices in India, ${ }^{\circ} 2$, p. 442.

Translation problem, $n^{\circ} 2$, p. 405.

Translations of Indian texts, $n^{\circ} 2$, p. 416.

Translators and interpreters in Puerto Rico, ${ }^{\circ} 4$, p. 652.
Translators in Quebec, $n^{\circ} 4$, p. 656.

Trivedi, Harish: India, England, France: A (Post-) Colonial Translational Triangle, $\mathrm{n}^{\circ} 2, \mathrm{p} .407$.

True equivalents, $n^{\circ} 1$, p. 124.

Untranslatable terms, $n^{\circ} 2$, p. 427.

Untranslated terms, $\mathrm{n}^{\circ} 2$, p. 427

V. Prasad, G. J. and N. Kamala: Trans-creating India(s): The Nation in English Translation, $\mathrm{n}^{\circ} 2$, p. 450 .

Women's writing in India, $\mathrm{n}^{\circ} 2$, p. 451.

Working memory, ${ }^{\circ} 4$, p. 624 